
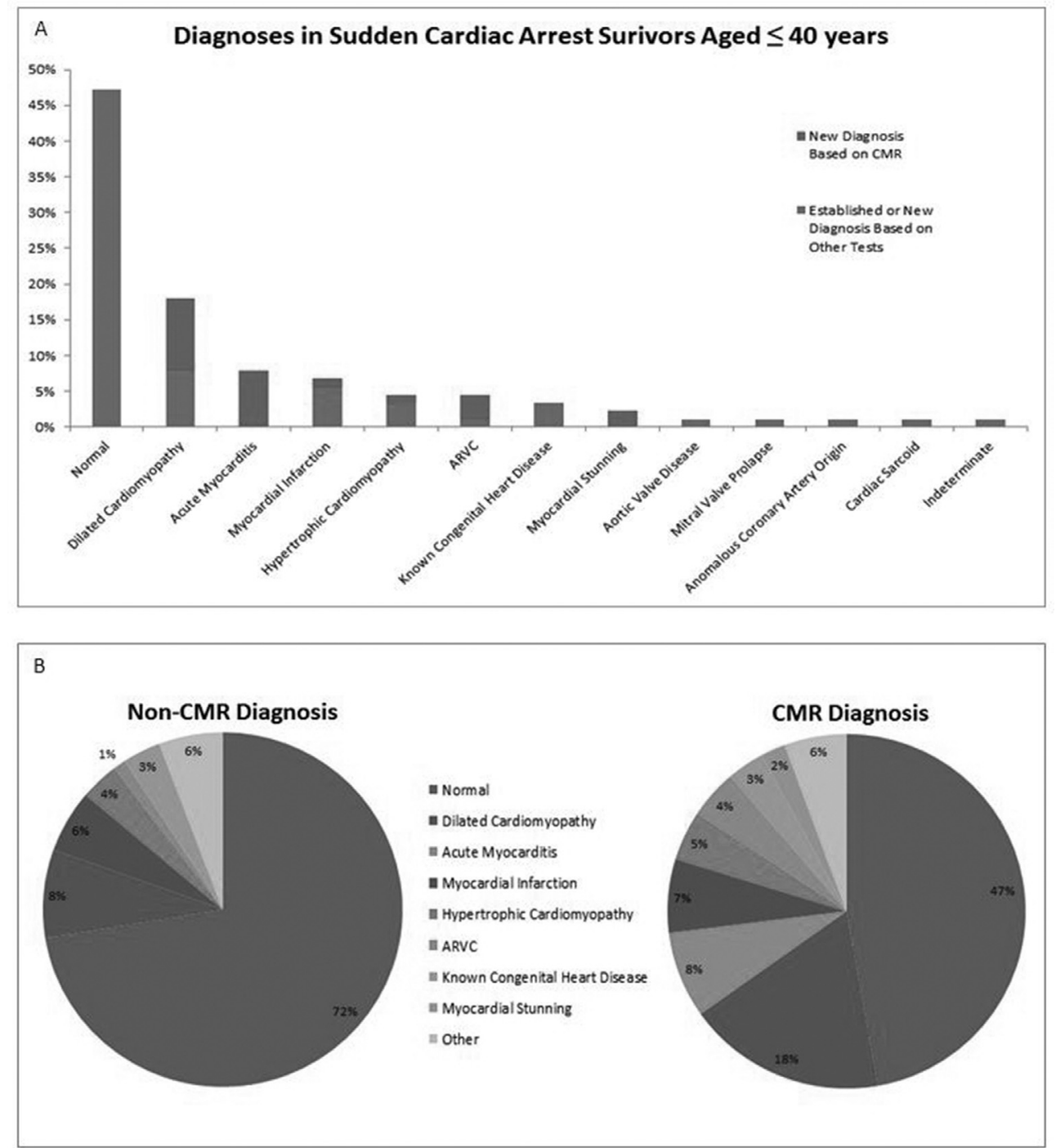

Abstract 50 Figure 1 Diagonses in survivors of suddem cardiac arrest aged $<40$ years (A), further subdivided into non-CMR diagnosis and CMR diagnosis (B). The subgroup classified as 'other' is composed of the diagnoses listed in figure A with a frequency of $<2 \%$ in this cohort.

\section{OUTCOMES WITH SINGLE-COIL VERSUS DUAL-COIL IMPLANTABLE CARDIOVERTER-DEFIBRILLATORS: A META-ANALYSIS}

Nicholas Sunderland*, Amit Kaura, Francis Murgatroyd, Para Dhillon, Paul Scott. King's College Hospital

\subsection{6/heartjnl-2017-311726.50}

Aims Dual-coil implantable cardioverter defibrillator (ICD) leads have traditionally been used over single-coil leads due to concerns regarding high defibrillation thresholds (DFT) and consequent poor shock efficacy. However, accumulating evidence suggests that this position may be unfounded and that dual-coil leads may also be associated with higher complication rates during lead extraction. This meta-analysis collates data comparing dual- and single-coil ICD leads.

Methods and results Electronic databases were systematically searched for randomised controlled trials (RCT) and non-randomised studies comparing single-coil and dual-coil leads. The mean differences in DFT and summary estimates of the odds- ratio (OR) for first-shock efficacy and the hazard-ratio (HR) for all-cause mortality were calculated using random effects models. Eighteen studies including a total of 138124 patients were identified. Dual-coil leads were associated with a lower DFT compared to single coil leads (mean difference $-0.83 \mathrm{~J}$; $95 \%$ confidence interval $[\mathrm{CI}]-1.39--0.27 ; \mathrm{p}=0.004)$. There was no difference in the first-shock success rate with dual-coil compared to single-coil leads (OR 0.74; 95\% CI 0.45-1.21; $\mathrm{p}=0.22$ ). There was a significantly lower risk of all-cause mortality associated with single-coil leads (HR 0.91; 95\% CI 0.86-0.95; p<0.0001).

Conclusion This meta-analysis suggests that single-coil leads have a marginally higher DFT but that this may be clinically insignificant as there appears to be no difference in first-shock efficacy when compared to dual-coil leads. The mortality benefit with single-coil leads most likely represents patient selection bias. Given the increased risk and complexity of extracting dual-coil leads, centres should strongly consider single-coil ICD leads as the lead of choice for routine new leftsided ICD implants. 